\title{
Sensibilidad antimicrobiana en aislamientos de líquido peritoneal de niños intervenidos por abdomen agudo e infección intraabdominal
}

\author{
Antimicrobial sensitivity in peritoneal fluid isolates of children taken \\ to surgery for acute abdomen and intra-abdominal infection
}

Sandra Jaqueline Beltrán ${ }^{1}$, Melissa Cruz², Eddy Carolina Pedraza ${ }^{2}$, Fredy Orlando Mendivelso ${ }^{3}$

Médica, infectóloga pediatra, Clínica Pediátrica Colsanitas, Bogotá, D.C., Colombia

Médica, residente de Pediatría, Fundación Universitaria Sanitas, Clínica Universitaria Colombia, Bogotá, D.C., Colombia

Médico cirujano, MPH, MSc, FETP, Clínica Reina Sofía, Bogotá, D.C., Colombia

\section{Resumen}

Introducción. La apendicitis aguda es causa frecuente de infección intraabdominal en pediatría. La elección del antibiótico se basa en los hallazgos quirúrgicos macroscópicos, el criterio médico, las guías locales o internacionales y, en pocas ocasiones, en el resultado de los aislamientos bacterianos en cultivos de líquido peritoneal y las tasas locales de resistencia. Se analizaron la frecuencia y la sensibilidad microbiológica de los cultivos de muestras de líquido peritoneal de pacientes de un mes a i6 años de edad con manejo quirúrgico por abdomen agudo con sospecha de infección intraabdominal.

Materiales y métodos. Se llevó a cabo un estudio prospectivo de cohorte con análisis de las historias clínicas y de resultados de laboratorio de niños sometidos a cirugía por abdomen agudo, en la que el cirujano tomó muestra del líquido peritoneal para la tipificación y determinación de los perfiles de sensibilidad de los microorganismos aislados. Los datos se analizaron con el programa Stata ${ }^{\mathrm{TM}}$, versión I5.0.

Resultados. Se identificaron 303 casos, de los cuales el 93,6 \% recibió profilaxis antibiótica con ampicilina-sulbactam y clindamicina-amikacina. El 95,3\% de los procedimientos fueron apendicectomías. Se tomó cultivo del $50 \%$ de las apendicitis perforadas. Se aislaron 48 microorganismos; el más frecuente (2,7 \%) fue Escherichia coli positiva para BLEE. El Ioo \% de los microorganismos resultaron ser sensibles a la amikacina, el $97,2 \%$ al meropenem, y el 94,4\%, a la ciprofloxacina, el cefepime y el ceftazidime. La resistencia más frecuente (37,I \%) fue contra la combinación de ampicilina y sulbactam, en los gérmenes Gram negativos.

Discusión. La evaluación de la sensibilidad antimicrobiana de los aislamientos de líquido peritoneal de los procedimientos quirúrgicos en pediatría, es una buena práctica clínica que orienta al cirujano en la adecuada selección del esquema antibiótico y, además, disminuye el riesgo de falla terapéutica temprana y la posibilidad

Fecha de recibido: 15/05/2019 - Fecha aceptación: 16/09/2019

Correspondencia: Fredy Orlando Mendivelso, Calle 127 N²0-78, Clínica Reina Sofía, Bogotá, D.C., Colombia. Teléfono: (304) $384-4102$. Correo electrónico: fmendivelso@colsanitas.com

Citar como: Beltrán SJ, Cruz M, Pedraza EC, Mendivelso FO. Sensibilidad antimicrobiana en aislamientos de líquido peritoneal de niños intervenidos por abdomen agudo e infección intraabdominal. Rev Colomb Cir. 2019;34:354-63. https://doi.org/10.30944/20117582.523

Este es un artículo de acceso abierto bajo una Licencia Creative Commons - BY-NC-ND https://creativecommons.org/licenses/by-nc-nd/4.0/deed.es 
de mayor resistencia o complicaciones infecciosas.

Palabras clave: antibióticos; farmacorresistencia microbiana; peritoneo; abdomen agudo; pediatría; procedimientos quirúrgicos.

\begin{abstract}
Introduction: Acute appendicitis is a cause of intra-abdominal infection in pediatrics. The choice of antibiotic is based on the macroscopic surgical findings, the medical criteria, the local guidelines and the result of the bacterial isolation in the peritoneal fluid and the local resistance rate. We aim to analyze the frequency and microbiological susceptibility in cultures performed in peritoneal fluid samples in patients from I month to I6 years with surgical management of acute abdomen with suspected intra-abdominal infection.
\end{abstract}

Materials and Methods: Prospective cohort study with analysis of clinical histories and laboratories in children taken to surgery for acute abdomen where the surgeon took a sample of peritoneal fluid for typing and determine susceptibility profiles of isolated microorganisms. Data analyzed with Stata V.i5.o.

Results: We identified 303 cases of which $93.6 \%$ received antibiotic prophylaxis with ampicillin/sulbactam and clindamycin-amikacin. $95.3 \%$ of the procedures were appendectomies. Cultivation was taken in 50\% of perforated appendicitis. Isolate 48 microorganisms, the most frequent Escherichia coli BLEE (+) (2.7\%). The $100 \%$ of the microorganisms were susceptible to amikacin, meropenem (97.2\%), ciprofloxacin, cefepime and ceftazidime (94.4\%). The highest resistance was presented with ampicillin/sulbactam (37.I\%) for Gram-negative organisms.

Conclusions: To evaluate the antimicrobial susceptibility in peritoneal fluid isolations in surgical procedures in pediatrics is a good clinical practice that is oriented in the surgeon in the adequate selection of an antibiotic scheme, the risk of early therapeutic failure and the possibility of greater resistance or complications infectious.

Key words: antibiotics; drug resistance, microbial; peritoneum; abdomen, acute; pediatrics; surgical procedures.

\section{Introducción}

Las infecciones intraabdominales generalmente son secundarias a la perforación o a la inflamación de la pared intestinal, a partir de la flora gastrointestinal habitual. Clínicamente, las infecciones intraabdominales se dividen en no complicadas -el proceso infeccioso se limita al órgano o tejido de origen- y complicadas, cuando la infección se extiende y afecta el peritoneo y produce peritonitis generalizada o procesos localizados como abscesos, con cuadros clínicos que pueden ir desde fiebre, anorexia y dolor, a sepsis y falla orgánica múltiple, choque séptico y muerte. Por tanto, la elección adecuada del tratamiento antimicrobiano se convierte en uno de los puntos primordiales del abordaje de las infecciones intraabdominales ${ }^{\mathrm{I}-3}$. Las enfermedades primarias que conducen a peritonitis varían según la región geográfica y la edad del paciente.

En los Estados Unidos e Israel, la apendicitis aguda es responsable de $82 \%$ y $92 \%$ de los casos, respectivamente ${ }^{4,5}$, mientras que, en África, es más frecuente la perforación intestinal secundaria a fiebre tifoidea, ascaridiasis, trauma o invaginación y, luego, la apendicitis aguda, siendo de suma importancia en esta región la enterocolitis necrosante ${ }^{6 .}$ La mortalidad atribuible a la peritonitis en los países desarrollados es del o,06 $\%$ 7; en África, varía entre el o,9 y el $2 \%$, lo que refleja, básicamente, las diferencias en el acceso a los servicios quirúrgicos y a las unidades de cuidado crítico ${ }^{8}$.

Las infecciones intraabdominales raramente 
son el resultado de un solo agente microbiano, y no es fácil el aislamiento de todos los microorganismos involucrados con los métodos rutinarios de laboratorio. Aunque pueden predecirse los microorganismos causantes de las infecciones intraabdominales adquiridas en la comunidad con base en la microbiota, su tasa de resistencia difiere según la zona geográfica, las comorbilidades y la exposición previa a antimicrobianos ${ }^{9-\mathrm{II}}$.

Desde el punto de vista clínico y terapéutico, vale la pena destacar exclusivamente dos condiciones de la enfermedad apendicular: la apendicitis no perforada y la perforada; esta última se caracteriza por el hallazgo de perforación en la exploración quirúrgica o en el reporte de histopatología ${ }^{9}$. La apendicitis perforada-cirugía contaminada- requiere tratamiento adicional con antibióticos hasta la resolución del cuadro clínico, lo que se asocia con mayor estancia hospitalaria, a diferencia de las apendicitis no complicadas o no perforadas, en las cuales solo se usan los antimicrobianos como profilaxis y, por ende, se suspenden luego del procedimiento quirúrgico ${ }^{12,13}$.

La mayoría de los reportes en la literatura mencionan que la flora encontrada en los aislamientos de líquido abdominal es mixta. Se destacan microorganismos como: Escherichia coli, Streptococcus milleri, Pseudomonas aeruginosa y Enterococcus spp.; entre los anaerobios, Bacteroides fragilis es el más frecuentemente aislado. Sin embargo, ni los microorganismos ni su resistencia a los antibióticos se consideran para indicar el manejo profiláctico o terapéutico de las infecciones intraabdominales, por el desconocimiento de la prevalencia y de la resistencia de los microorganismos a nivel local ${ }^{14}$.

Varios autores han reportado tasas de resistencia a la amoxicilina-clavulanato mayores de $40 \%$ en los aislamientos de líquido peritoneal, factor que, sumado a la falta de cobertura para microorganismos como $P$. aeruginosa, tercer bacteria en frecuencia, hace necesario replantear la utilidad de los esquemas empíricos profilácticos y de los esquemas terapéuticos utilizados en la apendicitis aguda y rescata la importancia de hacer los cultivos de líquido peritoneal, los cuales han sido abandonados en favor de abordajes rutinarios en estas circunstancias ${ }^{15,16}$.

Se ha descrito que, al menos, el 35,3\% de los niños sometidos a apendicectomía tienen cultivos positivos y, aunque muchos estudios reportan preferencias por el tratamiento profiláctico empírico de amplio espectro sobre el basado en la toma de cultivos del líquido peritoneal, esta decisión ignora las ventajas de los esquemas reducidos y ajustados, con menor impacto en la microbiota y en el riesgo de diarrea asociada a Clostridium difficile como infección emergente en los pacientes pediátricos ${ }^{17-20}$.

Entre los factores más asociados a las infecciones del sitio operatorio, está la duración de los síntomas preoperatorios ( $\geq 6$ horas), la perforación visceral, la técnica quirúrgica empleada y la elección del esquema antibiótico ${ }^{21-23}$. La introducción de nuevos antibióticos, el incremento de las tasas locales de resistencia a los antimicrobianos, la frecuencia de uso de los esquemas profilácticos y terapéuticos basados en la monoterapia o los tratamientos combinados para las infecciones intraabdominales en la población pediátrica, requieren el conocimiento de la epidemiología local con el fin de optimizar el uso de la terapia antimicrobiana ${ }^{24,25}$.

Son escasos en la literatura médica los datos locales sobre sensibilidad y resistencia a los antibióticos en la población pediátrica sometida a procedimientos quirúrgicos, en comparación con los reportados en la población adulta ${ }^{26,27}$. Los estudios de prevalencia en portadores nasofaríngeos reportan Staphylococcus aureus como el principal microorganismo aislado en el personal de salud de los hospitales pediátricos $(43, \mathrm{I} \%$ en el personal de enfermería y $23,5 \%$ en los médicos) con resistencia a los betalactámicos ${ }^{28}$. En el 2012, los hospitales pediátricos chilenos reportaron diferentes patrones de sensibilidad in vitro de E. coli en hemocultivos, relacionados con antibióticos de uso frecuente como la ampicilina (3I,9\%), la gentamicina $(89,9 \%)$, la cefalotina $(48,3 \%)$ y la cefotaxima $(88,6 \%)$. La menor sensibilidad in vitro para Klebsiella pneumoniae se presentó con 
la cefotaxima (62,6 \%), para $P$. aeruginosa, con el imipenem $(72,9 \%)$, y para Acinetobacter baumannii, con ampicilina-sulbactam $\left(67,6 \%{ }^{29}\right.$.

El propósito del estudio fue analizar la frecuencia y la sensibilidad microbiológica de los cultivos practicados a las muestras de la cavidad abdominal tomadas por sospecha de infecciones intraabdominales, en niños que requirieron manejo quirúrgico en la Clínica Universitaria Colombia y Clínica Reina Sofía de Bogotá, Colombia.

\section{Materiales y métodos}

Se llevó a cabo un estudio prospectivo de cohorte con muestreo consecutivo ${ }^{30}$. Se recolectó la información demográfica, los datos clínicos y los reportes de laboratorio de las historias clínicas y de las notas quirúrgicas de los niños entre un mes y I6 años de edad sometidos a cirugía con sospecha diagnóstica de abdomen agudo y con hallazgos operatorios de infección en la cavidad abdominal.

Los cirujanos pediatras tomaron muestras del líquido peritoneal para hacer los cultivos y antibiograma. La recolección de la información se hizo durante 12 meses de forma sistemática, de septiembre de 2015 a septiembre de 2016.

\section{Toma del cultivo}

En los niños con evidencia de infección en la cavidad abdominal durante la exploración quirúrgica, se tomó por aspiración una muestra del líquido durante el acto quirúrgico y se envió en jeringa estéril con capuchón sin retirar la aguja, idealmente, previa inoculación de la muestra en la botella de hemocultivos para anaerobios. Se inoculó la muestra en cantidad de 5 a Io $\mathrm{ml}$ (volumen mínimo de $5 \mathrm{ml}$ ) en esta botella de hemocultivo (relación I:Io ml). En el Laboratorio de Microbiología, se inoculó la muestra de líquido peritoneal en agar sangre al $5 \%$, MacConkey y caldo de enriquecimiento de tioglicolato, y se incubó por 48 horas en atmósfera aerobia, con lectura diaria de las cajas de agar. Se identificaron todos los microorganismos y su sensibilidad, mediante el método automatizado de Vitek $2 \mathrm{C}^{\mathrm{TM}}$ (BioMerieux).

\section{Análisis estadístico}

Se trabajó con el universo de los datos y las unidades de observación se recolectaron de forma consecutiva, previa validación y cumplimiento de los criterios de inclusión.

Se estimaron las medidas de frecuencia y de tendencia central para las variables continuas, y se evaluó la función de distribución de normalidad de las variables de interés mediante los gráficos P-P y Q-Q, y la prueba de Shapiro-Wilk. Las variables categóricas se analizaron mediante las pruebas no paramétricas de ji al cuadrado y exacta de Fisher. La comparación de las medias para muestras independientes se llevó a cabo con la prueba de la suma de rangos de Wilcoxon. Los valores de p para el contraste de hipótesis se consideraron estadísticamente significativos cuando p era mayor de 0,05. Los datos se analizaron con el software licenciado Stata ${ }^{\mathrm{TM}}$, versiónI5.0.

\section{Resultados}

Se analizó la información de 303 pacientes pediátricos con sospecha de abdomen agudo durante el periodo de estudio, es decir, de septiembre de 2015 a septiembre de 2016 , de los cuales 298 $(98,3 \%)$ presentaron hallazgos sugestivos de infección intraabdominal.

La mayoría (59 \%) de los pacientes eran del sexo masculino y los grupos más representados ( $82 \%)$ fueron los escolares y los adolescentes. El $94 \%$ no manifestaron antecedentes de consumo de antibióticos antes de la cirugía ( $\leq 48$ horas), mientras que el $4,3 \%$ restante tenía antecedentes de alguna enfermedad respiratoria que justificaba el manejo antibiótico previo (amigdalitis e infección respiratoria aguda, entre otras) (tabla I). El $93 \%$ recibió profilaxis con antibióticos antes del procedimiento quirúrgico y en el $6 \%$ no fue formulada desde urgencias por tratarse de una urgencia vital. El esquema antibiótico más utilizado (75\%) fue ampicilina-sulbactam, seguido de clindamicina-amikacina y, en menos casos, se utilizaron otros tratamientos combinados, como metronidazol-amikacina, $u$ otros poco convencionales como, por ejemplo, monoterapias (amikacina o ampicilina o clindamicina o cefazolina) 
Tabla 1. Características basales de los pacientes incluidos en el estudio

\begin{tabular}{lrr}
\hline \multicolumn{1}{c}{ Variable } & \multicolumn{2}{c}{ Valor } \\
\hline Pacientes pediátricos & \\
Sexo & & $180(59,4)$ \\
Masculino, n (\%) & & $123(40,6)$ \\
Femenino, n (\%) & $\mathbf{n ~ ( \% )}$ & \multicolumn{1}{c}{ IC $_{95 \%}$} \\
\hline Grupos de edad (años) & & \\
\hline Neonato & $2(0,7)$ & $(0,1-2,3)$ \\
Lactante menor & $5(1,7)$ & $(0,7-3,8)$ \\
Lactante mayor & $47(15,5)$ & $(11,8-20,0)$ \\
Preescolar & $115(38,0)$ & $(32,6-43,5)$ \\
Escolares & $134(44,2)$ & $(38,7-49,8)$ \\
Adolescencia & & \\
Consumo de antibiótico antes de la cirugía & \\
(sin que fuera profilaxis) & & $(2,5-7,2)$ \\
Sí & $287(94,7)$ & $(92,7-97,4)$ \\
No & $3(1,0)$ & $(0,1-2,9)$ \\
Sin información & & \\
\hline & &
\end{tabular}

o asociaciones como metronidazol-piperacilina-tazobactam (tabla 2).

El tiempo medio entre el diagnóstico clínico y el inicio de la cirugía fue de seis horas; sin embargo, fue de 240 horas en un paciente con abdomen agudo secundario a un absceso intraabdominal manejado inicialmente con antibióticos parenterales y drenaje guiado por radiología intervencionista, pero que requirió cirugía abierta por el deterioro clínico.

De los pacientes con diagnóstico de abdomen agudo, el 95,3\% correspondió a apendicectomía $\mathrm{y}$, los procedimientos restantes, a drenaje de colecciones, laparoscopia diagnóstica por obstrucción intestinal, intususcepción y colecistectomía, entre otros. La técnica más utilizada ( $75 \%$ ) fue la laparoscópica y solo un caso requirió conversión de la técnica a laparotomía ${ }^{31}$. El $72 \%$ de los cirujanos clasificó los procedimientos como cirugía limpia (tabla 3). Entre las descripciones quirúrgicas, se evidenció gran variabilidad en el reporte de los hallazgos del procedimiento descrito por los médicos tratantes (figura I).
Tabla 2. Antibióticos usados en la profilaxis de la cirugía

\begin{tabular}{lcc}
\hline \multirow{2}{*}{ Variable } & \multicolumn{2}{c}{ Valor } \\
\cline { 2 - 3 } & $\mathbf{n}(\%)$ & IC $_{95 \%}$ \\
\hline Uso de antibiótico profiláctico & \\
Sí & $280(92,4)$ & $(90,2-95,8)$ \\
No & $19(6,3)$ & $(4,10-9,71)$ \\
Sin información & $4(1,3)$ & $(0,1-5,0)$ \\
Número de dosis de antibiótico profiláctico & \\
Una & $257(84,8)$ & $(81,5-89,4)$ \\
Dos & $21(6,9)$ & $(4,6-10,5)$ \\
Ninguna & $19(6,3)$ & $(4,1-9,7)$ \\
Tres & $2(0,7)$ & $(0,1-2,4)$ \\
Sin información & $4(1,3)$ & $(0,1-5,0)$ \\
Esquema de antibióticos usados en profilaxis \\
Ampicilina-sulbactam & $228(75,2)$ & $(71,12-80,73)$ \\
Clindamicina más & $41(13,5)$ & $(10,27-18,07)$ \\
amikacina & $19(6,3)$ & $(4,10-9,71)$ \\
No se aplica & $2(0,7)$ & $(0,18-2,40)$ \\
Ampicilina & $2(0,7)$ & $(0,18-2,40)$ \\
Amikacina & $2(0,7)$ & $(0,18-2,40)$ \\
Piperacilina- & $1(0,3)$ & $(0,05-1,87)$ \\
tazobactam & $1(0,3)$ & $(0,05-1,87)$ \\
Clindamicina & & $(0,05-1,87)$ \\
Cefazolina & & $(0,1-5,0)$ \\
Amikacina más & & \\
metronidazol & & \\
Metronidazol más & & \\
tazobactindam información & & \\
\hline
\end{tabular}

De 285 pacientes con apendicectomía, el $83 \%$ correspondió a apéndices no perforadas, de las cuales únicamente se tomaron seis cultivos que representaban el 2,5\% de las no perforadas con seis aislamientos de E. coli. De 48 pacientes con diagnóstico de apendicitis perforada, se tomaron 24 (50 \%) cultivos, todos positivos, de los cuales I2 fueron tomados de pacientes con apendicitis clasificadas como gangrenosas; un cultivo correspondió a un apéndice que no se había clasificado como tal en la historia clínica y otros dos cultivos 
procedieron de pacientes sometidos a laparotomía por obstrucción intestinal, con un total de 33 (Io,9\%) tomas de cultivo de pacientes operados por sospecha de infección intraabdominal . Los cultivos analizados revelaron que el microorganismo aislado más frecuentemente fue $E$. coli (66\%); sin embargo, es importante destacar que el $54 \%$ de los aislamientos fueron polimicrobianos, con predominio ( $75 \%$ ) de los microorganismos

Tabla 3. Procedimiento quirúrgico

\begin{tabular}{|c|c|c|}
\hline \multirow[b]{2}{*}{$\begin{array}{l}\text { Tiempo entre el diagnóstico y la } \\
\text { cirugía (horas) }\end{array}$} & \multicolumn{2}{|c|}{ Valor } \\
\hline & & \\
\hline Media - Mediana & & $8,3-6$ \\
\hline Rango & & $0,50-240$ \\
\hline Procedimiento quirúrgico & n (\%) & $\mathrm{IC}_{95 \%}$ \\
\hline Apendicectomía & $267(88,1)$ & $(85,6-92,5)$ \\
\hline $\begin{array}{l}\text { Apendicectomía más drenaje } \\
\text { de peritonitis }\end{array}$ & $13(4,3)$ & $(2,5-7,3)$ \\
\hline $\begin{array}{l}\text { Laparoscopia diagnóstica } \\
\text { por obstrucción intestinal }\end{array}$ & $5(1,7)$ & $(0,7-3,8)$ \\
\hline Drenaje de peritonitis & $3(1,0)$ & $(0,3-2,9)$ \\
\hline Colecistectomía & $3(1,0)$ & $(0,3-2,9)$ \\
\hline $\begin{array}{l}\text { Laparoscopia diagnóstica } \\
\text { por intususcepción }\end{array}$ & $2(0,7)$ & $(0,1-2,4)$ \\
\hline $\begin{array}{l}\text { Apendicectomía más drenaje } \\
\text { por absceso apendicular }\end{array}$ & $2(0,7)$ & $(0,1-2,4)$ \\
\hline Drenaje de colección & $1(0,3)$ & $(0,0-1,8)$ \\
\hline $\begin{array}{l}\text { Apendicectomía más drenaje } \\
\text { de colección }\end{array}$ & $1(0,3)$ & $(0,0-1,8)$ \\
\hline $\begin{array}{l}\text { Apendicectomía más } \\
\text { laparoscopia diagnóstica por } \\
\text { peritonitis primaria }\end{array}$ & $1(0,3)$ & $(0,0-1,8)$ \\
\hline Sin información & $5(1,7)$ & $(0,7-3,8)$ \\
\hline \multicolumn{3}{|l|}{ Técnica quirúrgica } \\
\hline Laparoscópica & $230(75,9)$ & $(68,9-79,0)$ \\
\hline Abierta & $72(23,8)$ & $(20,6-30,7)$ \\
\hline Conversión & $1(0,3)$ & $(0,0-1,9)$ \\
\hline \multicolumn{3}{|c|}{ Clasificación de la cirugía según nota quirúrgica } \\
\hline Limpia & $221(72,9)$ & $(74,3-83,8)$ \\
\hline Sucia & $24(7,9)$ & $(5,8-12,5)$ \\
\hline Contaminada & $17(5,6)$ & $(3,8-9,5)$ \\
\hline Limpia-contaminada & $16(5,3)$ & $(3,5-9,1)$ \\
\hline Sin información & $25(8,3)$ & $(5,7-12,5)$ \\
\hline
\end{tabular}

Gram negativos (tabla 4). En el 2,7 \% de los microorganismos Gram negativos, se identificaron mecanismos de producción de betalactamasas de espectro extendido (positivos para BLEE).

Por otra parte, en el Laboratorio de Microbiología, se procesaron los cultivos para la búsqueda de anaerobios en las muestras de I4 pacientes con apendicitis perforada y se logró identificar microorganismos como Clostridium spp. y Prevotella spp. En los antibiogramas, todos (IO0 \%) los gérmenes Gram negativos resultaron ser sensibles a la amikacina, el 97,2\%, al meropenem, y el 94,4\%,

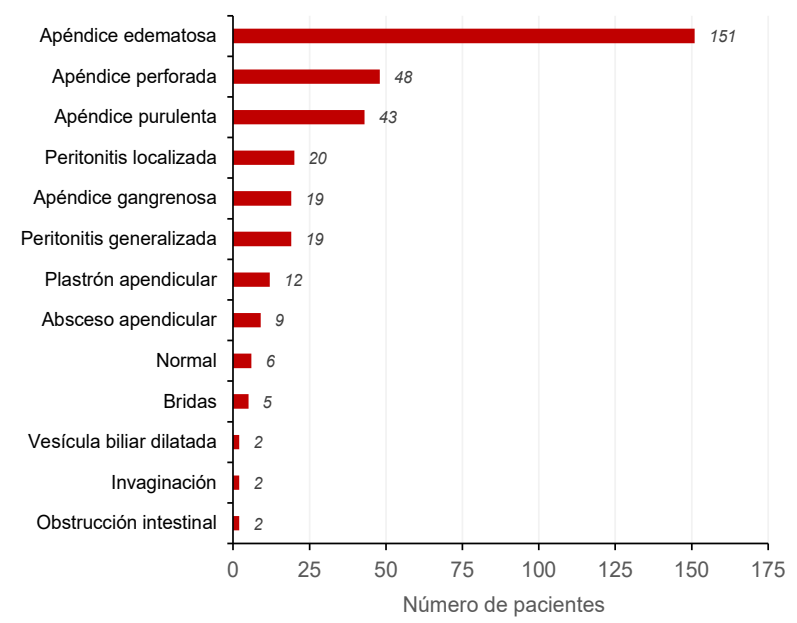

Figura 1. Descripción de hallazgos macroscópicos en cirugía

Tabla 4. Frecuencia de aislamientos microbiológicos en cultivos de líquido peritoneal

\begin{tabular}{lcc}
\hline \multicolumn{1}{c}{ Microorganismo } & $\mathbf{n}$ & (\%) \\
\hline Escherichia coli & 32 & 66,7 \\
Streptococcus viridans & 3 & 6,3 \\
Enterococcus faecium & 2 & 4,2 \\
Pseudomonas aeruginosa & 2 & 4,2 \\
Streptococcus constellatus & 2 & 4,2 \\
Enterococcus avium & 1 & 2,1 \\
Enterococcus faecalis & 1 & 2,1 \\
Enterococcus gallinarum & 1 & 2,1 \\
Klebsiella spp. & 1 & 2,1 \\
Raoultella ornithilolytica & 1 & 2,1 \\
Streptococcus anginosus & 1 & 2,1 \\
Streptococcus mitis & 1 & 2,1 \\
\hline
\end{tabular}


a la ciprofloxacina, el cefepime y el ceftazidime (figura 2). Los mayores porcentajes de resistencia para en los Gram negativos -incluidos E. coli, Klebsiella spp. y Pseudomonas spp.- se registraron con ampicilina-sulbactam (37\%), seguido por la gentamicina $(8,33 \%)$. En el caso de antibióticos con cobertura para los microorganismos Gram positivos, los mayores porcentajes (83\%) de sensibilidad reportados correspondieron a la vancomicina y la ampicilina, y el $58 \%$, a la eritromicina (figura 3).

\section{Discusión}

Esta investigación corresponde al primer trabajo conjunto entre cirujanos e infectólogos pediatras interesados en caracterizar la flora bacteriana y el perfil de sensibilidad a los antibióticos en los pacientes pediátricos sometidos a cirugía por abdomen agudo y sospecha de infección intraabdominal ${ }^{32}$. La apendicitis aguda fue causa del

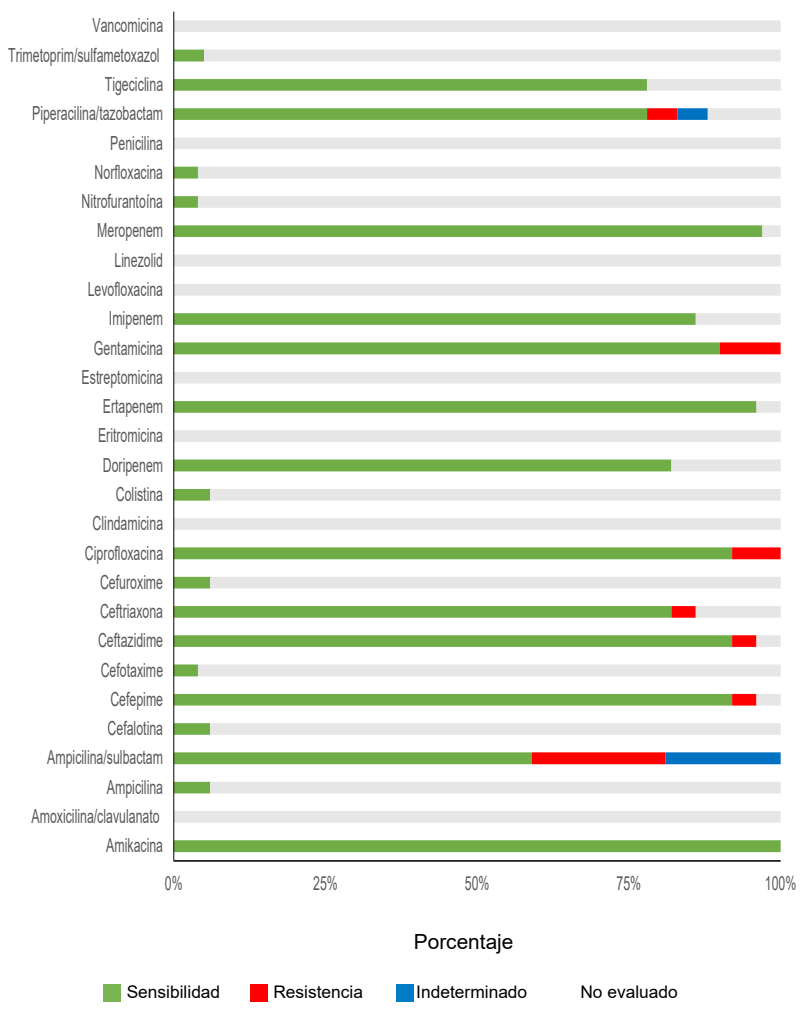

Figura 2. Perfil de sensibilidad y resistencia en cultivos realizados en niños con infección intraabdominal
$90 \%$ de las infecciones intraabdominales en la edad pediátrica, y el germen más prevalente (66 $\%)$ fue $E$. coli, seguido en menor grado por otros aerobios Gram positivos y P. aeruginosa; esto es similar a lo reportado en otros estudios en los cuales también reportan a E. coli como el principal agente patógeno, seguido de Klebsiella spp., Acinetobacter spp. y P. aeruginosa ${ }^{9,33,34}$.

Dado el papel primordial en nuestro medio de E. coli en la apendicitis perforada y como causa principal de peritonitis secundaria, el perfil de resistencia de este agente patógeno juega un papel fundamental, tanto en el tratamiento antimicrobiano profiláctico, como en la terapéutica seleccionada. Los datos demostraron resistencia intermedia en $\mathrm{I} 6 \%$ y, total, en $19 \%$ de los cultivos

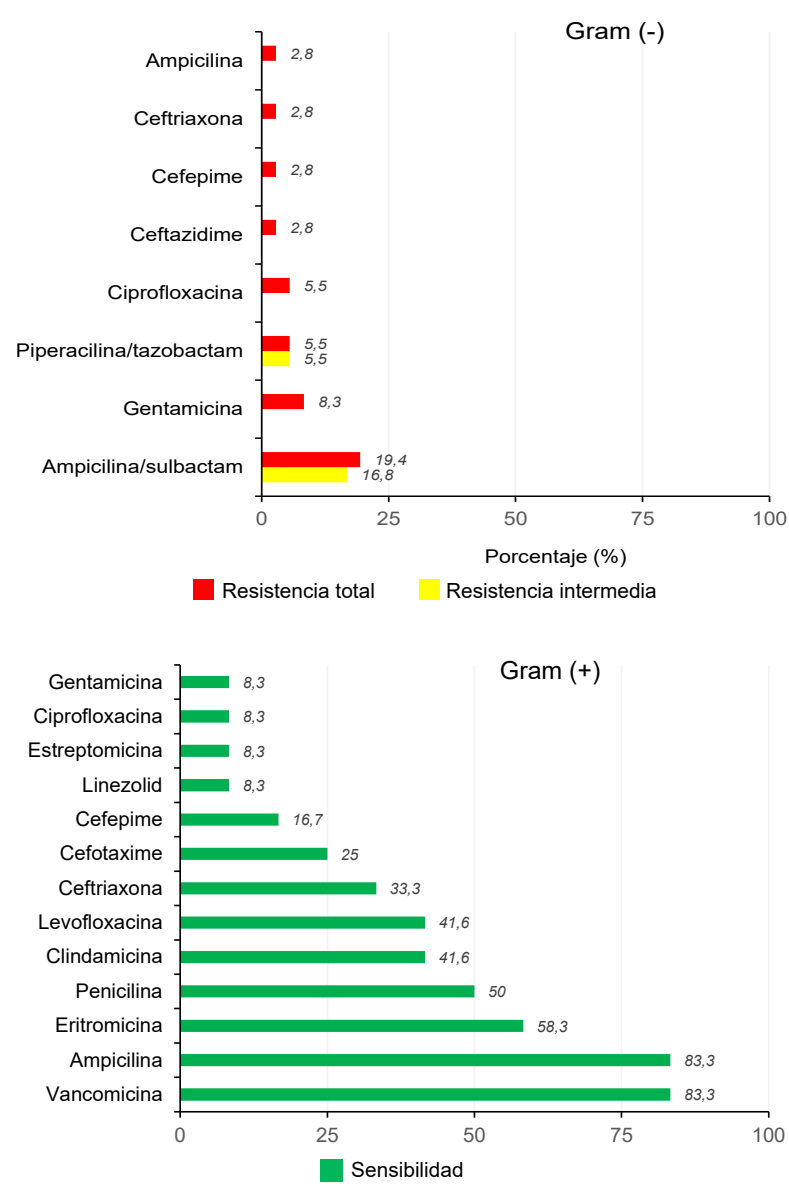

Figura 3. Perfil de resistencia y sensibilidad en microorganismos Gram negativos y Gram positivos aislados de cultivos de líquido peritoneal de niños sometidos a cirugía por abdomen agudo con sospecha de infección intraabdominal 
de E. coli ante la ampicilina-sulbactam, lo cual convierte este esquema antibiótico en una opción poco adecuada para la profilaxis y para el manejo terapéutico en presencia de infecciones intraabdominales, dado que puede incrementar el riesgo de infección posoperatoria ${ }^{35-37}$. A pesar del aumento de la prevalencia de gérmenes Gram negativos positivos para BLEE reportada a nivel mundial, en el presente estudio, la proporción de gérmenes positivos para BLEE fue baja (2,7 \%), cifras que se pueden explicar por la implementación del programa racional de optimización de los antibióticos que orienta el uso empírico y la duración de los esquemas antimicrobianos de menor espectro, guardando opciones como el carbapenem o la piperacilina-tazobactam solo para infecciones intraabdominales complicadas ${ }^{38}$.

A pesar del bajo porcentaje de muestras procesadas para anaerobios, los resultados refuerzan la conducta de la cobertura empírica que ahorra un recurso económico al no procesar estas muestras en condiciones anaerobias, ya sea por su pobre resultado o por la dificultad de que las muestras sean enviadas en condiciones óptimas de anaerobiosis desde las salas de cirugía ${ }^{33}$. Por otra parte, la clasificación de la apendicitis aguda como perforada o no perforada con base en los hallazgos quirúrgicos, debería ser uno de los objetivos próximos por considerar, tanto por los cirujanos pediatras como por los patólogos, reevaluando la clasificación actual de apéndice edematosa, gangrenosa o purulenta, ya que esta es la base con la cual se toman decisiones de manejo antimicrobiano, pronóstico y seguimiento del paciente ${ }^{39}$.

Dentro de la unificación de conceptos por parte del equipo quirúrgico, es de suma importancia establecer la adecuada clasificación de la herida y la intervención practicada a cada paciente, resaltando que tanto el manejo antibiótico como la asociación con la infección del sitio operatorio difieren según la situación clínica y el hallazgo o la ausencia de perforación apendicular ${ }^{40-42}$.

En muchas de las instituciones de salud del país, la elección del antibiótico para la infección intraabdominal depende de la decisión del cirujano o de la disponibilidad de los antimicrobianos; sin embargo, la recomendación de profilaxis o terapéutica debe ser guiada por datos objetivos, tanto de resistencia como de epidemiología local, lo cual favorecerá resultados más costo-efectivos para el paciente y para la institución.

La participación de los cirujanos pediatras en un programa racional de optimización de uso de los antibióticos, mejora la atención de los niños que consultan a los servicios de urgencias con una afección quirúrgica abdominal aguda ${ }^{43,44}$. Los cultivos rutinarios resultan ser de gran valor en la detección de los cambios epidemiológicos y de los patrones de resistencia a los antimicrobianos asociados con la peritonitis, lo que permite el ajuste subsecuente del tratamiento, según los patrones de sensibilidad y la evolución clínica.

Los esquemas antimicrobianos varían en cada institución, pero, en general, deben tener cobertura adecuada frente a enterobacterias y Bacteroides spp., y ser iniciado lo más pronto posible después del diagnóstico de infección intraabdominal, especialmente, en presencia de sepsis o choque séptico.

Según la Infectious Diseases Society of America (IDSA), entre los esquemas de tratamiento antibiótico más recomendados para los pacientes pediátricos con infecciones intraabdominales complicadas, se incluyen los esquemas basados en una de las siguientes opciones: un aminoglucósido, un carbapenem (imipenem, meropenem o ertapenem), un $\beta$-lactámico o un inhibidor de betalactamasas (piperacilina-tazobactam o ticarcilina-clavulanato), o una cefalosporina de generación avanzada (cefotaxime, ceftriaxona, ceftazidima o cefepima) con metronidazol, dejando el uso de clindamicina sujeto a las tasas de resistencia de $B$. fragilis. Sin embargo, no se discute el impacto sobre la flora microbiana ni la opción de tener tratamientos dirigidos con un menor impacto ecológico, que sean eficaces y con cobertura adecuada para la flora implicada, y que contribuyan igualmente a un menor número de complicaciones infecciosas posoperatorias ${ }^{44,45}$.

En conclusión, el conocimiento de la resistencia y de la epidemiología nos permite tratar 
los pacientes con infección intraabdominal no complicada con esquemas antimicrobianos de espectro limitado, disminuyendo la toxicidad y los efectos colaterales sin repercusión en el éxito terapéutico, lo cual hace de esta terapia antimicrobiana dirigida una opción costo-beneficiosa que, al igual que en otras publicaciones, no conlleva un aumento de las complicaciones posoperatorias cuando se compara con un esquema de mayor espectro.

\section{Cumplimiento de normas éticas}

Consentimiento informado: El protocolo de investigación contó con la evaluación y aprobación de las comisiones de investigación y ética de las dos instituciones participantes en el estudio. Se obtuvieron los consentimientos informados y se encuentran disponibles para quienes los requieran.

Conflictos de interés: ninguno declarado

Fuente de financiación: recursos propios de los investigadores

\section{Referencias}

I. García-Sánchez JE, García-García MI, García-Garrote F, Sánchez-Romero I. Diagnóstico microbiológico de las infecciones intraabdominales. Enferm Infecc Microbiol Clin. 2013;31:230-9. https:doi.org/IO.IOI6/j. eimc.2012.0I.023

2. Coccolini F, D'Amico G, Sartelli M, Catena F, Montori $\mathrm{G}$, Ceresoli $\mathrm{M}$, et al. Antibiotic resistance evaluation and clinical analysis of acute appendicitis. Report of I,43I consecutive worldwide patients: A cohort study. Int J Surg. 20I6;26:6-II. doi: IO.IOI6/j.ijsu.20I5.I2.063.

3. Shirah GR, O'Neill PJ. Intra-abdominal Infections. Surg Clin North Am. 20I4;94:I319-33. doi:IO.IOI6/j. suc.2014.08.005.

4. Long SS, Pickering LK, Prober CG. Principles and practice of pediatric infectious diseases. Philadelphia: Elsevier Health Sciences; 2012. p. 82-90. ISBN: 9781437727029.

5. Gofrit ON, Abu-Dalu K. Perforated appendicitis in the child: Contemporary experience. Isr Med Assoc J. 200I;3:262-5. doi: I0.I0I6/j.jpedsurg.2008.05.013.

6. Brender JD, Marcuse EK, Koepsell TD, Hatch EI. Childhood appendicitis: Factors associated with perforation. Pediatrics. 1985;76:30I-6.

7. Serres SK, Cameron DB, Glass CC, Graham DA, Zurakowski D, Karki M, et al. Time to appendectomy and risk of complicated appendicitis and adverse outcomes in children. JAMA Pediatr. 20I7;I71:740-6. doi: I0.IOoI/ jamapediatrics.20I7.0885.

8. Adesunkanmi ARK, Oseni SA, Adejuyigbe O, Agbakwuru EA. Acute generalized peritonitis in African children: Assessment of severity of illness using modified APACHE II score. ANZ J Surg. 2003;73:275-9.

9. Hadley GP. Intra-abdominal sepsis --epidemiology, aetiology and management. Semin Pediatr Surg. 20I4;23:357-62. doi:I0.I053/j.sempedsurg.20I4.06.0o8.

Io. Blot S, De Waele JJ. Critical issues in the clinical management of complicated intra-abdominal infections. Drugs. 2005;65:I6II-20. doi: I0.2165/00003495$200565120-00002$.

II. Huang XZ, Zhu LB, Li ZR, Lin J. Bacterial colonization and intestinal mucosal barrier development. World J Clin Pediatr. 2013;2:46-53. doi:I0.5409/wjcp.v2.i4.46.

I2. Emil S, Gaied F, Lo A, Laberge JM, Puligandla P, Shaw $\mathrm{K}$, et al. Gangrenous appendicitis in children: A prospective evaluation of definition, bacteriology, histopathology, and outcomes. J Surg Res. 20I2;I77:123-6. doi:I0.IOI6/j.jss.2012.03.0I0.

I3. Bansal S, Banever GT, Karrer FM, Partrick DA. Appendicitis in children less than 5 years old: Influence of age on presentation and outcome. Am J Surg. 2012;204:I03I5. doi:IO.IOI6/j.amjsurg.2OI.

I4. Boueil A, Guegan H, Colot J, D’Ortenzio E, Guerrier G. Peritoneal fluid culture and antibiotic treatment in patients with perforated appendicitis in a Pacific Island. Asian J Surg. 2015;38:242-6. doi:IO.IoI6/j.asjsur.2015.03.005.

15. Chan KWE, Lee KH, Mou JWC, Cheung ST, Sihoe JDY, Tam YH. Evidence-based adjustment of antibiotic in pediatric complicated appendicitis in the era of antibiotic resistance. Pediatr Surg Int. 2010;26:157-60. doi: I0.1007/s00383-009-2540-6.

I6. Dahlberg M, Almstrom M, Wester T, Svensson JF. Intraoperative cultures during appendectomy in children are poor predictors of pathogens and resistance patterns in cultures from postoperative abscesses. Pediatr Surg Int. 2019;35:34I-6. doi: I0.IOO7/soo383-0I8-04428-3.

I7. Ibieta MF, Castaño IM, Ríos PR, Perotti KC, Ticona JR, Vallejo OG, et al. Estudio de la flora patógena y resistencias en apendicitis pediátricas. Cir Pediatr. 20I4;27:16-20.

I8. Castagnola E, Bandettini R, Ginocchio F, Perotti M, Masa DL, Ciucci A, et al. Susceptibility to antibiotics of aerobic bacteria isolated from community acquired secondary peritonitis in children: Therapeutic guidelines might not always fit with and everyday experience. J Chemother. 2013;25:213-6. doi: I0.II79/1973947813Y.ooo 0000083.

19. Shree N, Arora BS, Mohil RS, Kasana D, Biswal I. Bacterial profile and patterns of antimicrobial drug resistance in intra-abdominal infections: Current experience in a teaching hospital. Indian J Pathol Microbiol. 2013;56:388-92. doi: I0.4IO3/0377-4929.I2532I. 
20. Guillet-Caruba C, Cheikhelard A, Guillet M, Bille E, Descamps P, Yin L, et al. Bacteriologic epidemiology and empirical treatment of pediatric complicated appendicitis. Diagn Microbiol Infect Dis. 2011;69:3768I. doi: I0.IOI6/j.diagmicrobio.20I0.II.003.

2I. Eisenberg D. Surgical site infections: Time to modify the wound classification system? J Surg Res. 2012;175:545. doi: I0.IOI6/j.jss.20II.07.025.

22. Teixeira PG, Sivrikoz E, Inaba K, Talving P, Lam L, Demetriades D. Appendectomy timing: Waiting until the next morning increases the risk of surgical site infections. Ann Surg. 2012;256:538-43. doi: IO.IO97/SLA. obor3e3i8265ear3.

23. Rafiq MS, Khan MM, Khan A, Jan H. Evaluation of postoperative antibiotics after non-perforated appendectomy. J Pak Med Assoc. 2015;65:815-7.

24. Fallon SC, Hassan SF, Larimer EL, Rodríguez JR, Brandt ML, Wesson DE, et al. Modification of an evidence-based protocol for advanced appendicitis in children. J Surg Res. 2013;I85:273-7. doi: IO.IOI6/j.jss.2013.05.088.

25. Badal RE, Bouchillon SK, Lob SH, Hackel MA, Hawser SP, Hoban DJ. Etiology, extended-spectrum beta-lactamase rates and antimicrobial susceptibility of Gram-negative bacilli causing intra-abdominal infections in patients in general pediatric and pediatric intensive care units--global data from the Study for Monitoring. Pediatr Infect Dis J. 2013;32:636-40. doi: I0.I097/INF.oboi3e3I82886377.

26. Scapellato PG, Pessacq P, Corso A, Pasteran F, Rapoport $\mathrm{M}$, Vasen $\mathrm{W}$, et al. Etiología aerobia de apendicitis aguda en adultos. Estudio multicentrico de la sepsis abdominal en argentina. Medicina (B. Aires). 20I7;77:I2I-4.

27. Morganti L, Córdova E, Cassini E, Gómez N, López LM, Badia M, et al. Sensiblidad antimicrobiana de bacilos gramnegativos de infecciones intraabdominales de la comunidad en un hospital de la ciudad de Buenos Aires, Argentina. Rev Española Quimioter. 20I6;29:202-5.

28. González-Martínez ML, Hernández-Castellanos NB, Cruz-Betancour E, Hernández-Hernández Y, Medina-Mauri R. Susceptibilidad antimicrobiana de Staphylococcus aureus en trabajadores de un hospital pediátrico. Rev Ciencias Médicas Pinar del Río. 2018;22:15-24.

29. Cifuentes M, Silva F, García P, Bello H, Briceño I, Calvo $\mathrm{M}$, et al. Susceptibilidad antimicrobiana en Chile 2012. Revista Chilena de infectología. 20I4;3I:123-30.

30. Rodríguez M, Mendivelso F. Diseño de investigación de corte transversal. Rev Médica Sanitas. 2018;2I:I4I-6. doi: I0.26852/0I234250.20.

3I. García GA, Jiménez G, Barrios AJ, Guevara RE, Ruiz JP, Mendivelso FO. El cambio del paradigma educativo en la enseñanza de la cirugía laparoscópica. Rev Colomb Cirugía. 2017;32:40-4. doi: I0.30944/20II7582.6.

32. Bonilla E. La cirugía pediátrica, una obra de caridad. Rev Colomb Cirugía. 20I3;28:266-70.

33. Brook I. Microbiology and management of intra-abdominal infections in children. Pediatr Int. 2003;45:123-9.
34. Newman N, Wattad E, Greenberg D, Peled N, Cohen Z, Leibovitz E. Community-acquired complicated intra-abdominal infections in children hospitalized during 1995-2004 at a paediatric surgery department. Scand J Infect Dis. 2009;4I:720-6. doi: I0.1080/0036554090315926I.

35. Boomer LA, Cooper JN, Deans KJ, Minneci PC, Leonhart K, Diefenbach KA, et al. Does delay in appendectomy affect surgical site infection in children with appendicitis? J Pediatr Surg. 2014;49:IO26-9. doi: IO.IOI6/j.jpedsurg.2.

36. Obinwa O, Casidy M, Flynn J. The microbiology of bacterial peritonitis due to appendicitis in children. Ir J Med Sci. 20I4;183:585-9I. doi: I0.IOO7/sII845-OI3-I055-2.

37. Alverdy JC, Hyoju SK, Weigerinck M, Gilbert JA. The gut microbiome and the mechanism of surgical infection. Br J Surg. 2017;IO4:eI4-e23. doi: I0.IO02/bjs.IO405.

38. Shawyer AC, Hatchell AC, Pemberton J, Flageole H. Compliance with published recommendations for postoperative antibiotic management of children with appendicitis: A chart audit. J Pediatr Surg. 2015;50:783-5. doi: I0.IOI6/j.jpedsurg.2015.02.040.

39. Zinn JL. Surgical wound classification: Communication is needed for accuracy. AORN J. 20I2;95:274-8. doi: IO.IOI6/j.aorn.20II.IO.0I3.

40. Lee SL, Islam S, Cassidy LD, Abdullah F, Arca MJ. Antibiotics and appendicitis in the pediatric population: An American Pediatric Surgical Association Outcomes and Clinical Trials Committee systematic review. J Pediatr Surg. 20I0;45:2I8I-5. doi: I0.IOI6/j.jpedsurg.20I0.06.038.

4I. Ortega G, Rhee DS, Papandria DJ, Yang J, Ibrahim AM, Shore $\mathrm{AD}$, et al. An evaluation of surgical site infections by wound classification system using the ACS-NSQIP. J Surg Res. 20I2;I74:33-8. doi: IO.IOI6/j.jss.20II.05.056.

42. Yousef Y, Youssef F, Homsy M, Dinh T, Pandya K, Stagg $\mathrm{H}$, et al. Standardization of care for pediatric perforated appendicitis improves outcomes. J Pediatr Surg. 2017;52:1916-20. doi: I0.IOI6/j.jpedsurg.2017.08.054.

43. Liu J, Li N, Hao J, Li Y, Liu A, Wu Y, et al. Impact of the antibiotic stewardship program on prevention and control of surgical site infection during peri-operative clean surgery. Surg Infect (Larchmt). 20I8;19:326-3. doi: IO.IO89/sur.20I7.20I.

44. Solomkin JS, Mazuski JE, Bradley JS, Rodvold KA, Goldstein EJC, Baron EJ, et al. Diagnosis and management of complicated intra-abdominal infection in adults and children: Guidelines by the Surgical Infection Society and the Infectious Diseases Society of America. Surg Infect (Larchmt). 20I0;II:79-I09. doi: 0.I089/sur.2009.9930.

45. Hurst AL, Olson D, Somme S, Child J, Pyle L, Ranade D, et al. Once-daily ceftriaxone plus metronidazole versus ertapenem and/or cefoxitin for pediatric appendicitis. J Pediatric Infect Dis Soc. 20I7;6:57-64. doi: IO.I093/jpids/ pivo82. 